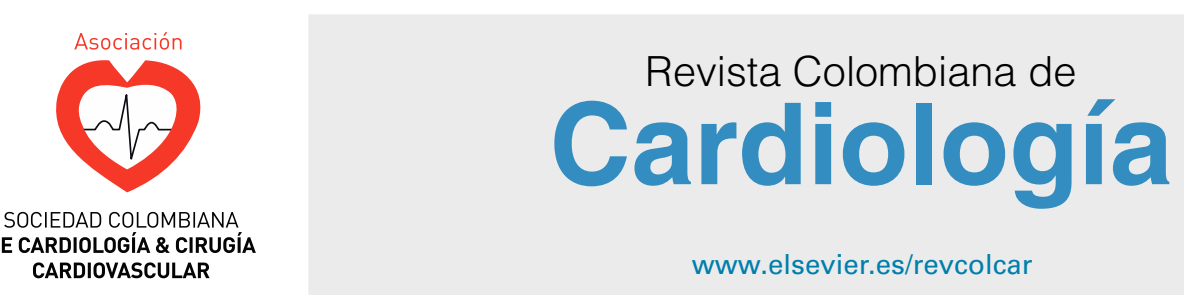

CARDIOLOGÍA DEL ADULTO - PRESENTACIÓN DE CASOS

\title{
Gran masa intracardiaca como causa de disnea y palpitaciones en mujer joven que acude a urgencias
}

\author{
Jerson Quitian Moreno ${ }^{\mathrm{a}, \mathrm{b}, *}$, Carlos Alberto Luengas Luengas ${ }^{\mathrm{b}}$, \\ María Juliana Rodríguez González ${ }^{a}$, Robinson Sánchez García ${ }^{a}$ \\ y Laura Milena Bermúdez López ${ }^{a}$ \\ a Cardiología Clínica, Fundación Cardiovascular de Colombia, Floridablanca, Colombia \\ ${ }^{\mathrm{b}}$ Métodos diagnósticos no Invasivos, Fundación Cardiovascular de Colombia, Floridablanca, Colombia
}

Recibido el 11 de junio de 2018; aceptado el 2 de abril de 2019

Disponible en Internet el 9 de julio de 2019

\author{
PALABRAS CLAVE \\ Tumor; \\ Disnea; \\ Palpitación; \\ Mixoma
}

\section{KEYWORDS}

Tumour;

Dyspnoea;

Palpitation;

Myxoma

\begin{abstract}
Resumen Los tumores cardíacos primarios son poco frecuentes y a menudo asintomáticos. Tienen una incidencia que varía de $0,0017 \%$ a $0,28 \%$. El diagnóstico diferencial de masas cardíacas incluye vegetaciones, trombos y tumores. Puede involucrar el endocardio, miocardio o epicardio. La afectación secundaria del corazón por tumores extracardíacos es rara. Los tumores benignos constituyen el $80 \%$ de las neoplasias cardíacas primarias y los mixomas son el tipo predominante. El tumor cardíaco maligno más frecuente es el angiosarcoma, y se caracteriza por crecimiento rápido, invasión local y metástasis a distancia. Se presenta el caso de paciente adulta joven con síntomas cardiovasculares inespecíficos asociados a pérdida de peso, cuyo diagnóstico ecocardiográfico e histopatológico es compatible con mixoma cardíaco.

( 2019 Sociedad Colombiana de Cardiología y Cirugía Cardiovascular. Publicado por Elsevier España, S.L.U. Este es un artículo Open Access bajo la licencia CC BY-NC-ND (http:// creativecommons.org/licenses/by-nc-nd/4.0/).
\end{abstract}

Large intra-cardiac mass as a cause of dyspnoea and palpitations in a young woman seen in an emergency department

Abstract Primary cardiac tumours are rare and are often asymptomatic, with an incidence that varies from $0.0017 \%$ to $0.28 \%$. The differential diagnosis of cardiac masses includes, growth, clots, and tumours. It can involve the endocardium, myocardium or epicardium. The secondary involvement of the heart due to extra-cardiac tumours is rare. Benign tumours make up $80 \%$ of the primary cardiac neoplasms, and myxomas are the most predominant type. The most common malignant cardiac tumour is the angiosarcoma, and is characterised by rapid growth,

\footnotetext{
* Autor para correspondencia.

Correo electrónico: jersonquitian@yahoo.com (J. Quitian Moreno).
} 
local invasion, and distant metastases. A case is presented of a young adult patient, with nonspecific cardiovascular symptoms together with a loss of weight. Her echocardiographic and histopathology diagnosis was compatible with a cardiac myxoma.

(c) 2019 Sociedad Colombiana de Cardiología y Cirugía Cardiovascular. Published by Elsevier España, S.L.U. This is an open access article under the CC BY-NC-ND license (http:// creativecommons.org/licenses/by-nc-nd/4.0/).

\section{Introducción}

Los tumores cardíacos primarios son poco frecuentes y a menudo asintomáticos, y poseen una incidencia que varía entre 0,0017\% a 0,28\% ${ }^{1}$. El diagnóstico diferencial de masas cardíacas incluye vegetaciones, trombos y tumores, y puede involucrar endocardio, miocardio o epicardio. La afectación secundaria del corazón por tumores extracardíacos es rara. Los tumores benignos constituyen el $80 \%$ de las neoplasias cardíacas primarias y los mixomas son el tipo predominante ${ }^{2}$. El tumor cardíaco maligno más frecuente es el angiosarcoma, que se caracteriza por crecimiento rápido, invasión local y metástasis a distancia ${ }^{3}$. Se presenta el caso de una paciente adulta joven, con síntomas cardiovasculares inespecíficos asociados a pérdida de peso, cuyo diagnóstico ecocardiográfico e histopatológico fue compatible con mixoma cardíaco.

\section{Caso}

Paciente de sexo femenino, de 27 años, quien consultó a diferentes centros de salud por síntomas principales de palpitaciones y disnea de moderados esfuerzos, asociados a pérdida de peso en los últimos dos meses; ingresó remitida a la institución, donde se logró mejoría de síntomas principales con dosis de betabloqueador endovenoso. Se solicitó ecocardiograma transtorácico en cuyo reporte se describió mixoma de gran tamaño en la aurícula izquierda, mamelonado, de aproximadamente $21 \mathrm{~cm}^{2}$, con fragmentos móviles que sugerían fácil embolización, oclusión del canal de la válvula mitral en aproximadamente un 90\%, adherido al septum posterosuperior con una base de implantación cercana a $1 \mathrm{~cm}$ de diámetro, y fracción de eyección del ventrículo izquierdo del 65\% (figs. 1 a y b).

En vista de lo anterior se indicó cirugía cardiovascular de urgencia, en la que se hizo resección de la masa sin complicaciones. El resultado de Patología correspondió a mixoma; en controles posteriores se observó evolución adecuada (fig. 2).

\section{Discusión}

El diagnóstico diferencial de lesiones intracavitarias incluye tumores benignos, malignos primarios, secundarios metastásicos y trombos. La lesión puede detectarse mediante ecocardiografía, en tanto que el diagnóstico diferencial debe incluir trombo, vegetación y cuerpo extraño.
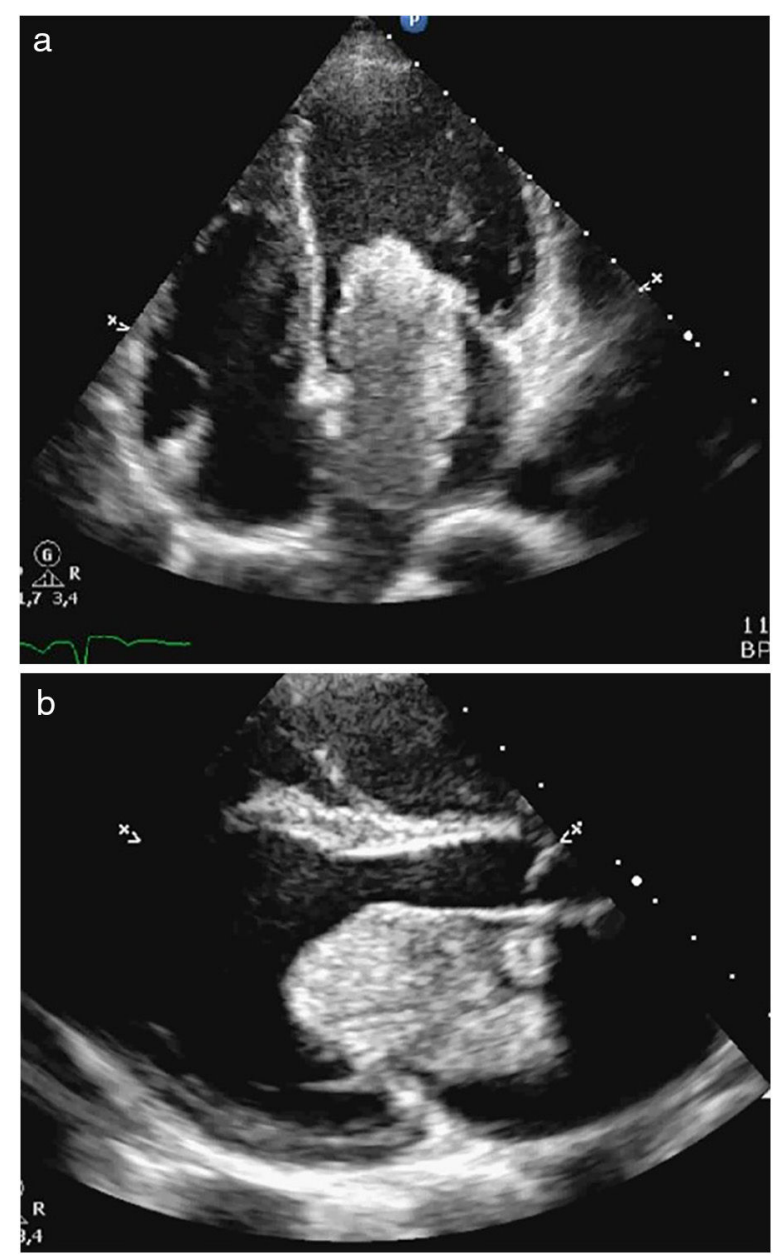

Figura 1 a y b Ecocardiografía transtorácica, que muestra masa bien definida en la aurícula izquierda, compatible con mixoma, que ocluye el tracto de entrada del ventrículo izquierdo en un 90\% (a: vista paraesternal eje largo; b: vista de cuatro cámaras).

Los tumores cardíacos representan el $0,2 \%$ de todos los tumores encontrados en humanos. Estos se dividen en primarios o secundarios/metastásicos. Los secundarios o metastásicos son 20 a 40 veces más frecuentes que los primarios; estos últimos son raros.

Aproximadamente, el $75 \%$ son benignos y cerca del $50 \%$ son mixomas, que tienen una incidencia de 0,0017\% en la población general. Los mixomas auriculares son los más comunes entre los tumores intracardiacos primarios del 


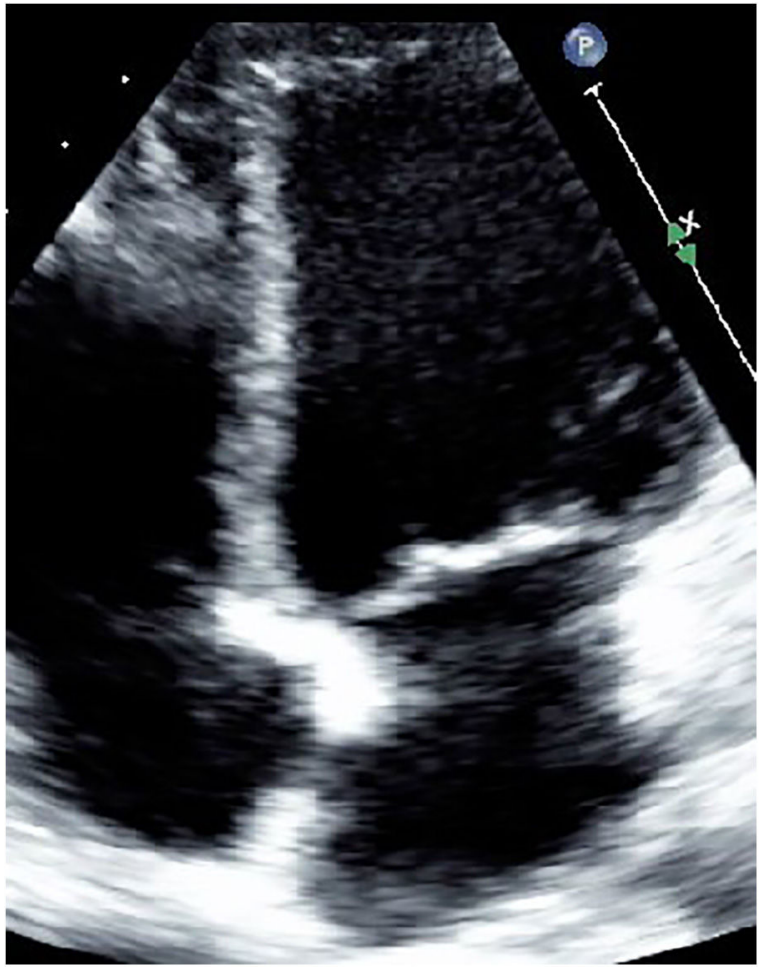

Figura 2 Ecocardiografía transtorácica que muestra imagen sin lesión residual (vista de cuatro cámaras).

adulto (aproximadamente 20 a $30 \%)^{3}$. Dos tercios de los mixomas surgen en la aurícula izquierda, mientras que otros nacen en la aurícula derecha, los ventrículos, la vena cava superior o las venas pulmonares ${ }^{4}$. Solo el 15 al $20 \%$ de los mixomas cardíacos se originan de la aurícula derecha.

La morfología típica del mixoma generalmente es polipoide, a menudo pedunculada, rara vez sésil, redonda u oval, con una superficie lisa o suavemente lobulada.

La tríada clásica encontrada en pacientes con mixoma cardíaco se caracteriza por la obstrucción del flujo sanguíneo, síntomas constitucionales y eventos tromboembólicos. La obstrucción del flujo sanguíneo conduce a insuficiencia cardíaca intermitente, además de malestar sistémico no específico de tipo gripal, generalmente hay fiebre baja, de larga duración, artralgia, anorexia y eventos tromboembólicos. Si la lesión se encuentra en el lado derecho el mixoma puede obstruir la válvula tricúspide, y causa signos y síntomas de insuficiencia cardíaca del lado derecho, edema periférico, ascitis, congestión hepática y síncope.

Los signos y síntomas provocados por los mixomas son atípicos y altamente variables, lo que puede dificultar el diagnóstico de esta neoplasia. De acuerdo con el tamaño, la movilidad y la ubicación del tumor, pueden tener un curso asintomático o presentar tantos síntomas como eventos tromboembólicos, que incluso pueden conducir a la muerte súbita; es por esto que se considera una urgencia cardiovascular, dado su potencial riesgo embólico, entre otras complicaciones $^{3,4}$.

\section{Conclusión}

El diagnóstico del mixoma cardíaco puede pasar inadvertido e incluso terminar en un diagnóstico erróneo, debido a su amplia variabilidad de síntomas; pese a ser considerado un tumor benigno y tener una incidencia baja en la población general, su localización intracardiaca implica un riesgo inminente para la vida al generar una serie de complicaciones que en algunos casos pueden incluso terminar en muerte súbita; debido a lo anterior es importante tomarlo siempre en cuenta dentro de los posibles diagnósticos en pacientes con alguna sintomatología correspondiente, garantizando de esta manera un diagnóstico ecocardiográfico temprano y un tratamiento efectivo y definitivo.

\section{Conflicto de intereses}

Ninguno.

\section{Bibliografía}

1. Ribeiro Dias R, Fernandes F, Alvarez Ramires FJ, Mady C, Piva Albuquerque C, Biscegli Jatene F. Mortality and embolic potential of cardiac tumors. Arq Bras Cardiol. 2014;103:13-8.

2. Oliveira R, Branco L, Galrinho A, Abreu A, Abreu J, Fiarresga A, et al. Cardiac myxoma: a 13-year experience in echocardiographic diagnosis. Rev Port Cardiol. 2010;29:1087-100.

3. Wei-Chieh Lee, Min-Ping Huang, Morgan Fu. Multiple intracardiac masses: myxoma, thrombus or metastasis: a case report. 2015;9:179-84

4. Vale Mde P, Freire Sobrinho A, Sales MV, Teixeira MM, Cabral KCh. Giant myxoma in the left atrium: case report. Rev Bras Cir Cardiovasc. 2008;23:276-8. 